\title{
Image Inpainting Using Patch Sparsity
}

\author{
Chetan Bhele ${ }^{1}$, Aslam Kazi ${ }^{2}$ \\ ${ }^{1,2}$ (Electronics Department, AISSMS College of Engineering Pune, India)
}

\begin{abstract}
In this paper we discuss a modified exemplar-based inpainting method through investigating the sparsity of natural image patches. In the exemplar-based algorithms, with the help of available information the unknown blocks of target region are inpainted by the most similar blocks extracted from the source region. To decide the filling order of missing pixels ensures the connectivity of object boundaries. The priority term shold defined. In the exemplar-based patch sparsity approaches, a sparse representation of missing pixels was considered to define a new priority term. Here, we discuss modification of the priority term and take measures to compute the similarities between fill-front and candidate patches. Image inpainting by patch propogation using patch sparsity shows the effeteness over traditional exemplar based inpainting.
\end{abstract}

Keywords: Image inpainting, texture synthesis, patch sparsity, patch propagation, sparse representation.

\section{Introduction}

The Reconstructing of missing region in an image, which is called image inpainting, is an important topic in the field of image processing. Applications of image inpainting include old film restoration, video inpainting [1], de-interlacing of video sequences [2], and cloud removal from remotely sensed images [3]. Many successful algorithms for image inpainting have been developed in the past decade which can be broadly categorized into the following groups:

1. Diffusion based algorithms.

2. Texture based algorithms.

3. Exemplar and search based algorithms

4. Sparsity based algorithms

The most fundamental inpainting approach is the diffusion based approach, in which the missing region is filled by diffusing the image information from the known region into the missing region at the pixel level. Within the category of PDE-based methods, there are a number of approaches which perform well for piecewise smooth images with sharp edges. These algorithms are well founded on the theory of partial differential equation (PDE) and variational method. Bertalmio et al. [4] filled in holes by continuously propagating the isophote (i.e., lines of equal gray values) into the missing region. They tend to introduce smooth effect in the textured region or larger missing region.

The second categories of approaches texture synthesis techniques were proposed. The common idea in these methods is to duplicate the information for the source region into the target region helps to fill large regions with pure textures hence, the texture information is preserved. Texture synthesis approaches are classified into pixel based sampling and patch-based sampling according to the sample texture size. Since the filling process in pixel-based schemes is being performed pixel by pixel, the algorithms are very slow. Although the speed of patch-based sampling was greatly improved, in which the target region is filled in by blocks of pixels, but discontinuous flaws between neighboring patches still remains.

Third category of approaches is the exemplar-based inpainting algorithm. This approach propagates the image information from the known region into the missing region at the patch level. This idea stems from the texture synthesis. However, natural images are composed of structures and textures, in which the structures constitute the primal sketches of an image (e.g., the edges, corners, etc.) and the textures are image regions with homogenous patterns or feature statistics (including the flat patterns). Criminisi et al. [5] designed an examplarbased inpainting algorithm by propagating the known patches into the missing patches gradually. To handle the missing region with composite textures and structures, patch priority is defined to encourage the filling-in of patches on the structure. $\mathrm{Wu}[6]$ proposed a cross-isophotes exemplar-based inpainting algorithm, in which a cross-isophotes patch priority term was designed based on the analysis of anisotropic diffusion. Wong [7] proposed a nonlocal means approach for the exemplar-based inpainting algorithm. The image patch is inferred by the nonlocal means of a set of candidate patches in the known region instead of a single best match patch. As compared with the diffusion-based inpainting algorithm, the exemplar-based inpainting algorithms have performed plausible results for inpainting the large missing region.

Recent approaches based on image sparse representation have also been introduced for the inpainting problem. In these methods, an image is presented by a sparse combination of an over complete set of transformations (e.g., wavelet, contourlet, DCT, etc.), and then the missing pixels are inferred by adaptively updating the sparse representation. Elad et al[8], proposed an approach to separate the image into cartoon 
(structure) and texture components, and then represented the sparse combination of the two obtained components by two incoherent over-complete transformations. This approach can effectively fill in the regions with structure and texture, it may fail to repair the structure or might produce smoothing. A sparse representation-based iterative algorithm for image inpainting proposed by Fadili et al [9]. They used the Expectation Maximization (EM) framework to consider that the missing samples can be recovered based on representations. $\mathrm{Xu}$ and Sun [10] suggested an exemplar-based inpainting method using a patch sparsity representation. They introduced the idea of sparse representation under the assumption that the missing patch could be represented by sparse linear combinations of candidate patches. Then, a constrained optimization model was proposed for the patch inpainting. Nonetheless, the edges in the filled regions sometimes are not connected properly. Hesabi and amir [11] proposed a modified patch propagation based image inpainting they define new priority term for sparse representation of missing pixel.

In this paper an extensive survey of previous work related to digital inpainting techniques will be presented. The survey includes scientific techniques used mainly in the research environment. Despite the significant differences between the existent techniques, in the authors manage to draw the main directions that each method must follow.

The remainder of our work is organized as follows. In Section 2, we explain the Exemplar-based image inpainting. In Section 3, we explain patch sparsity based image inpainting method. Section 4 we compare the results obtained from both methods. Finally Section 5, gives the concluding remarks.

\section{Exemplar Base Image Inpainting}

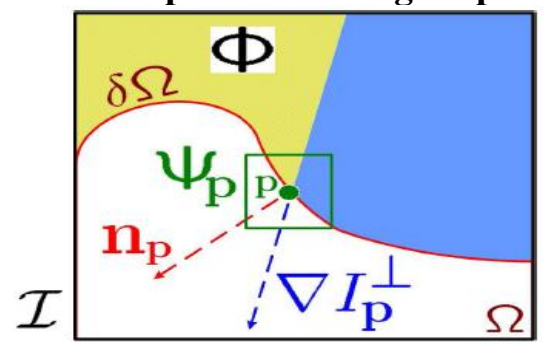

Fig. 1 Notation diagram

The exemplar based approach is an important class of inpainting algorithms. And they have proved to be very effective. Basically it consists of two basic steps: in the first step priority assignment is done and the second step consists of the selection of the best matching patch. The exemplar based approach samples the best matching patches from the known region, whose similarity is measured by certain metrics, and pastes into the target patches in the missing region. Exemplar- based Inpainting iteratively synthesizes the unknown region i. e. target region, by the most similar patch in the source region. According to the filling order, the method fills structures in the missing regions using spatial information of neighboring regions. This method is an efficient approach for reconstructing large target regions. Given an image I with the missing region $\Omega$ and the known Region $\Omega$, the task of image inpainting is to fill in the target Region using the image information in the source region $\delta \Omega$. The boundary of the target region is denoted by $\Psi_{p}$, which is called the fill-front in the exemplarbased inpainting algorithm. We further denote as a patch centered at a pixel .

Algorithm 1 : Exemplar base Image Inpainting

1: For each point $\mathrm{p}$ on the boundary $\delta \Omega$, a patch $\Psi_{p}$ is constructed, with $\mathrm{p}$ in the center of the patch.

2: Computing patch priorities: Given a patch $\Psi p$ centered at the point $\mathrm{p}$ for some $\mathrm{p} \epsilon \delta \Omega$. $\mathrm{P}(\mathrm{p})$ the priority value is defined as the product of two terms:

$\mathrm{P}(\mathrm{p})=\mathrm{C}(\mathrm{p}) . \mathrm{D}(\mathrm{p})$

Here, $C(p)$ the confidence term and $D(p)$ the data term, and they are defined as follows:

$C(p)=\frac{\Sigma C(q)_{i} q \in\left(\Psi_{p n \Omega)}\right.}{\left|\Psi_{p}\right|}, \quad D(p)=\frac{\left\|\nabla_{p}^{\mathbb{1}} n p\right\|}{a}$

where, $\left\|\Psi_{p}\right\|$ is the area of $\Psi_{p}, a$ is a normalization factor, np is a unit vector orthogonal to the boundary at the point $\mathrm{p}$, and $\nabla \frac{1}{p}$ is an isophote vector $\mathrm{D}(\mathrm{p})$ encourages linear structures to be synthesized first, and thus propagates securely into the target region, $\mathrm{C}(\mathrm{p})$ illustrates the amount of the reliable information surrounding the pixel $\mathrm{p}$ and is initialized to be

$\mathrm{C}(\mathrm{p})=0, \forall \mathrm{p} \in \Omega$, and $\mathrm{C}(\mathrm{p})=1, \forall \mathrm{p} \notin \Omega$ 
3: The patch $\Psi^{\prime} p^{\prime}$ with the highest priority is found to be filled in with the information extracted from the source region $\varphi$.

4: A global search is carried out on the whole image to find a patch $\Psi_{q}$ that has the most similarity with $\Psi_{p}^{\prime}$. Formally,

$\Psi_{q^{i}}=\arg \min \epsilon^{*} \phi\left(\Psi_{p^{\prime}}^{\prime} \Psi_{q}\right)$

where, the distance $d$ between two generic patches is simply defined as the sum of squared differences (SSDs) of the already known pixels in the two patches.

5: The value of each pixel to be filled in, $\mathrm{P}^{\prime} \mid \mathrm{p}^{\prime} \epsilon^{\prime}\left(\Psi_{p} \cap \Omega\right)$, is copied from its corresponding position inside $\Psi q$.

6: The confidence term $\mathrm{C}(\mathrm{p})$ is updated in the area encircled by $\Psi_{p}$ as follows:

$\mathrm{C}(\mathrm{q})=\mathrm{C}\left(\mathrm{p}^{\prime}\right), \forall \mathrm{q} \epsilon\left(\Psi_{p} \cap \Omega\right)$

The method investigates the sparsity of image patches, and measures the confidence of the patch located at the structure region by the sparseness of its nonzero similarities to the neighboring patches.

\section{Patch Sparsity Based Image Inpainting}

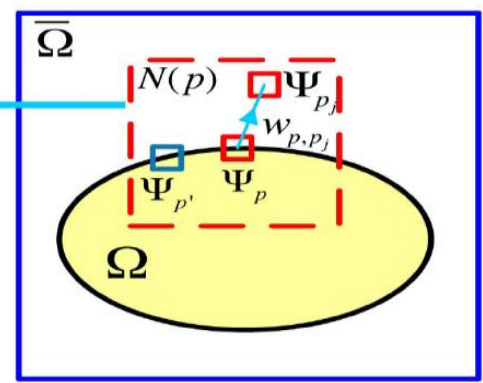

Fig 2: $\quad$ Patch selection

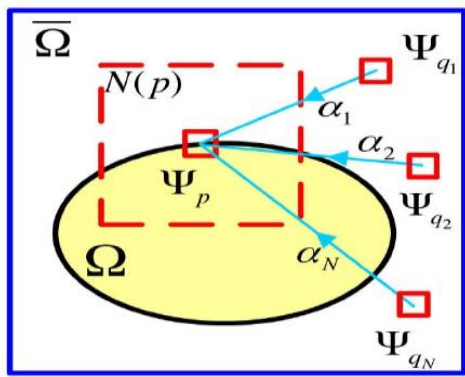

Patch Inpainting

The patch with larger structure sparsity is assigned a higher priority for further inpaintings. the steps 2 and 4 of Algorithm 1 are modified by $\mathrm{Xu}$ and sung[10] as follows:

2: A new definition for patch priority, structure sparsity, is proposed. For any selected patch, a collection of neighboring patches with highest similarities are also distributed in the same structure or texture. Therefore, the confidence of structure for a patch is measured by the sparseness of its nonzero similarities to the neighboring patches. The patch with more sparsely distributed nonzero similarities is laid on the fill-front due to the high structure sparseness. For the patch , located at the fill-front $\delta \Omega$, a neighborhood window $\mathrm{N}(\mathrm{p})$, with the center $\mathrm{p}$, is set. The sparseness of similarities for the patch is measured by

$P(p)=\sqrt{\left.\left[\sum_{p_{j} \in N s(p)} W^{2} \mathrm{p}, \mathrm{pj}\right]-4 a c\right] \frac{|N s(p)|}{|N(p)|}}$

where, the patch $\Psi p, p j$ is located in the known region centered at $P j . W p, p j$ belongs to the similarity between $\Psi_{p}$ and $\Psi_{p j}$, defined as:

$W p, p j=\frac{1}{z(p)} \exp \left(-\frac{\left(d \Psi_{p}, \Psi_{j}\right)}{\sigma^{2}}\right)$

with $d$ measuring the mean squared distance of the already known pixels in the two patches, $Z(p)$ being a normalization constant so that

$\sum_{p j \in \epsilon_{N s(p)}} W^{1}$ p, pj $=1$, and $\sigma$ being set to 5 . Finally, Ns(p) belongs to the set of thePj, that is,

$\mathrm{Ns}(\mathrm{p})=\left[\mathrm{Pj}: \mathrm{Pj} \in \mathrm{N}(\mathrm{p})\right.$ and $\left.\Psi p \in \subset \Omega^{\prime}\right]$

The patch priority (or structure sparsity) term is defined as the product of the transformed structure sparsity term and the patch confidence term:

$\mathrm{P}(\mathrm{p})=T_{\left[\xi_{1} 1\right]}(P(p)) \cdot C(p)$,

where, $T_{[\xi, 1]}$ is a linear transformation taking $\mathrm{P}(\mathrm{p})$ into the interval[$\xi$, 1]. This transformation scales the structure sparsity variations to be comparable with $\mathrm{C}(\mathrm{p})$.

4: In the patch sparsity inpainting method, $\Psi_{p}$ is inpainted by the sparse combinations of multiple exemplars in the framework of sparse representation. from the source region, the top $\mathrm{N}$ most similar patches, as the set of 
candidates , $\Psi q_{q=1}^{N}$ are selected. Therefore, the unknown pixels in patch $\Psi_{p}$ is approximated by linear combinations of the $\Psi_{q_{q=1}^{N}}^{N}$ ie.

$\Psi_{q}^{\prime}=\sum_{q}^{N} \alpha_{q} \Psi_{q}$

where, the coefficient $\bar{\alpha}=\left\{\alpha_{1}, \alpha_{2}, \alpha_{3}, \ldots \alpha_{N}\right\}$ is obtained by solving a constrained optimization problem in the framework of a sparse representation. This optimization problem minimizes the $l 0$ norm of $\bar{\alpha}$, i.e., the number of nonzero elements in the vector $\bar{\alpha}$, with the linearity assumption of the combination. The modified priority function defined by Samayeh Hesabi and Nezam Amiri[11] which is explain in Algoritham 2.

Algorithm 2 : A modified patch propagation-based image inpainting using patch sparsity

1: For each point $\mathrm{p}$ on the boundary $\delta \Omega$, a patch $\Psi_{p}$ is constructed, with $\mathrm{p}$ in the center of the patch.

2: To compute the patch's priority $\mathrm{P}(\mathrm{p})$, a stable definition is used:

$\mathrm{P}(\mathrm{p})=\alpha T_{[\xi, 1]}(P(p))+T_{[\gamma, 1]}(\mathrm{C}(\mathrm{p}))$

where, the terms $\alpha T_{[\xi, 1]}(P(p))$ and $\mathrm{C}(\mathrm{p})$ are the same as the ones defined in [10], and $\alpha$ and $\beta$ are the component weights with $0 \leq \alpha, \beta \leq 1$ and $\alpha+\beta=1$. As illustrated in [5], the confidence value rapidly drops to zero as the filling process goes on. When the dropping effect occurs, error continually propagates to the central part of the reconstructed image, causing noticeable visual artefacts. Therefore, a regularizing transformation is used to control the decreasing rate of the confidence term. In our experiments, we use a linear transformation T' to take $\mathrm{C}(\mathrm{p})$ into the interval $[\gamma, 1]$. Also, the priority term is changed to an additive form instead of a multiplicative form.

3: The patch $\Psi p^{\prime}$ with the highest priority is found to be filled in with the information extracted from the source region $\varphi$.

4: A global search is carried out on the whole image to find a patch $\Psi q$ that has the most similarity with $\Psi^{\prime}$. Formally,

$\Psi_{q^{\prime}}=\arg \min \epsilon \phi d\left(\Psi_{p^{\prime}}^{\prime} \Psi_{q}\right)$

where, the distance $d$ between two generic patches is simply defined as the sum of squared differences (SSDs) of the already known pixels in the two patches.

5: The value of each pixel to be filled in, $\mathrm{P}^{\prime} \mid \mathrm{p}^{\prime} \epsilon^{\prime}\left(\Psi_{p} \cap \Omega\right)$, is copied from its corresponding position inside $\Psi q$.

6: The confidence term $\mathrm{C}(\mathrm{p})$ is updated the area encircled by $\Psi^{\prime} p^{\prime}$ as follow

$\mathrm{C}(\mathrm{q})=C\left(\mathrm{p}^{\circ}\right) \frac{\left|\left\{q \mid q \in\left(\Psi_{p}{ }^{\circ} \cap \Omega\right)\right\}\right|}{\left|\Psi_{p^{s}}\right|} \forall \mathrm{q} \in\left(\Psi_{p^{\prime}} \cap \Omega\right)$

where, the numerator in the first term is number of known pixel in the patch $\Psi_{p^{\prime}}$.

\section{Comparisons}

As proposed in [11], we applied the conventional method with the patch size ( $\Psi p)$ being equal to $9 \times 9$ pixels. In proposed algorithm, the size of the patch window $\Psi$ could be set to $7 \times 7$ pixels and the size of the neighborhood window, $\mathrm{N}(\mathrm{P})$, Will set to $11 \times 11$ and the value of $\alpha$ and $\beta$ were set to 0.5 and 0.5 , respectively. Also, the number of candidate patches, $\mathrm{N}$, was fixed to be 21 for better results. The results obtained by our proposed algorithm. We find out that the results of our proposed algorithm are superior over the Past method. Also, the number of candidate patches, $\mathrm{N}$, were fixed to be 11,3 and 25 , correspondingly. The results obtained by our algorithm appear to be closer to the original image than the ones obtained by the other two methods. For a quantitative comparison, we computed the PSNR and SSIM values between the original and inpainted images, also observing better obtained PSNR and SSIM values by Algorithm 2.

\section{Conclusion}

In this paper, we have looked at three different types of inpainting methods. For each of the algorithms, we have reviewed and provided a detailed explanation of the process used for filling an obstruction making use of images and pseudo-code wherever appropriate. In addition, we have updated confidence value in adaptive way in both a qualitative and quantitative manner. From this analysis, a number of shortcomings and limitations were highlighted in relation to the type of information each algorithm can restore.

The exemplar-based inpainting algorithms have performed plausible results for inpainting the large missing region. But they work well only if the missing region consists of simple structure and texture. Thus we 
recommend the modified patch propagation based image inpainting using patch sparsity method for better image quality. It is capable of producing amazing results in reference to time. It takes minimum process time compared to exemplar based inpainting, also if it is applied major image blocks will not be lost and the final result will not have uneven features which are not pleasing to human eyes.

\section{Proceedings Papers:}

\section{References}

[1]. X. Li and Y. Zheng, "Patch-based video processing: A variational bayesian approach," IEEE Trans. Circuits Syst. Video Technol., vol. 19 , no. 1, pp. 27-40, Jan. 2009.

[2]. C. Ballester, M. Bertalmio, V. Caselles, L. Garrido, A. Marques, and F. Ranchin, "An inpainting-based deinterlacing method," IEEE Trans. Image Process., vol. 16, no. 10, pp. 2476-2491, Oct. 2007.

[3]. A. Maalouf, P. Carre, B. Augereau, and C. Fernandez Maloigne, "A bandelet-based inpainting technique for clouds removal from remotely sensed images," IEEE Trans. Geosci. Remote Sens., vol. 47, no. 7, pp. 2363-2371, Jul. 2009.

[4]. M. Bertalmio, L. Vese, G. Sapiro and S. Osher, "Simultaneous structure and texture image inpainting," IEEE Trans. Image Process, vol. 12, pp. 882-889, 2003.

[5]. A. Criminisi, P. Perez, and K. Toyama, "Object removal by examplarbased image inpainting," in Proc. Int. Conf. Comp. Vision, 2003, pp. 721-728.

[6]. J.Wu and Q. Ruan, "Object removal by cross isophotes examplar-based image inpainting," in Proc. Int. Conf. Pattern Recognition, 2006, pp. 810-813.

[7]. A. Wong and J. Orchard, "A nonlocal-means approach to examplarbased inpainting," presented at the IEEE Int. Conf. Image Processing, 2008.

[8]. M. Elad, J. L. Starck, P. Querre, and D. L. Donoho, "Simultaneous cartoon and texture image inpainting using morphological component analysis," Appl. Comput. Harmon. Anal., vol. 19, pp. 340-358, 2005.

[9]. M. J. Fadili, J. L. Starck and F. Murtagh, "Inpainting and zooming using sparse representations," The Comput. J., vol. 52, no. 1, pp. 64-79, 2009.

[10]. Z. Xu and J. Sun, "Image inpainting by patch propagation using patch sparsity," IEEE Transactions on Image Processing, vol. 19, no. 5 , pp. $1153-1165,2010$.

[11]. Samayeh Hesabi, Nezam Amiri, "Modified patch propogation-based image inpainting Using patch Sparsity,”AISP, 2012. 